Association for Information Systems AIS Electronic Library (AISeL)

Wirtschaftsinformatik Proceedings 2003

Wirtschaftsinformatik

September 2003

\title{
Wirkung von Patenten auf das Innovationsverhalten der Softwareindustrie
}

\author{
Norman Hoppen \\ Johann Wolfgang Goethe Universität Frankfurt am Main, hoppen@wiwi.uni-frankfurt.de \\ Daniel Beimborn \\ Johann Wolfgang Goethe Universität Frankfurt am Main \\ Wolfgang König \\ Johann Wolfgang Goethe Universität Frankfurt am Main, koenig@wiwi.uni-frankfurt.de
}

Follow this and additional works at: http://aisel.aisnet.org/wi2003

\section{Recommended Citation}

Hoppen, Norman; Beimborn, Daniel; and König, Wolfgang, "Wirkung von Patenten auf das Innovationsverhalten der Softwareindustrie" (2003). Wirtschaftsinformatik Proceedings 2003. 91.

http://aisel.aisnet.org/wi2003/91

This material is brought to you by the Wirtschaftsinformatik at AIS Electronic Library (AISeL). It has been accepted for inclusion in Wirtschaftsinformatik Proceedings 2003 by an authorized administrator of AIS Electronic Library (AISeL). For more information, please contact elibrary@aisnet.org. 
In: Uhr, Wolfgang, Esswein, Werner \& Schoop, Eric (Hg.) 2003. Wirtschaftsinformatik 2003: Medien - Märkte - Mobilität, 2 Bde. Heidelberg: Physica-Verlag

ISBN: 3-7908-0111-9 (Band 1)

ISBN: 3-7908-0116-X (Band 2)

(C) Physica-Verlag Heidelberg 2003 


\title{
Wirkung von Patenten auf das Innovations- verhalten der Softwareindustrie
}

\author{
Norman Hoppen, Daniel Beimborn, Wolfgang König \\ Johann Wolfgang Goethe Universität Frankfurt am Main
}

\begin{abstract}
Zusammenfassung: Neben der derzeit aktuellen juristischen Fragestellung, ob Computerprogramme den Anforderungen eines patentierfähigen Gutes entsprechen, gerät der ökonomische Zusammenhang zwischen Patenten und Innovationen ein wenig in den Hintergrund. Die These, dass Patente einen Anreiz schaffen, Innovationskosten auf sich zu nehmen, um diese durch positive Einnahmen zu kompensieren und zu sichern, ist aus mikroökonomischer Perspektive zumindest theoretisch unumstritten. Offen bleibt allerdings die Frage, ob aus makroökonomischer Sicht der Anreiz mögliche Blockadestrategien überkompensiert. Ziel des Artikels ist es, darzustellen, wie sich das Innovationsverhalten von Softwareentwicklern durch die Möglichkeit des Patentschutzes verändert. Zu diesem Zweck wurden ein ökonomisches Modell entwickelt und erste simulative Ergebnisse erzielt.
\end{abstract}

Schlüsselworte: Softwarepatente, Innovationen

\section{Einleitung}

Patente auf Computerprogramme (Software) sind derzeit in Deutschland ein stark diskutiertes Thema. Hintergrund ist zum einen die unterschiedliche Rechtslage in Deutschland und den USA, und zum anderen die aus ökonomischer Sicht umstrittene Wirkung von Patenten. Während in Deutschland Software grundsätzlich nicht dem Patentschutz unterliegt, sind der Patentierfähigkeit von Software in den USA gegenwärtig keine Grenzen gesetzt.

Trotz grundsätzlichem Patentierungsverbot von Software zeigt die Rechtspraxis in Deutschland, das schon seit längerem Patente auf Computerprogramme vergeben werden. Wolfgang Tauchert vom deutschen Patent- und Markenamt in München schätzt die Anzahl der im Jahr 2001 in Deutschland erteilten Patente, in denen Software involviert war, auf 500 [DPMA02].

In diesem Artikel wird ein ökonomisches Modell vorgestellt, das die wesentlichen Einflussfaktoren auf das Innovationsverhalten von Softwareentwicklern (Anbietern) formalisiert. In einem simulativen Ansatz wird das Innovationsverhalten mit 
Patentschutz und ohne Patenschutz analysiert. Hiermit wird das Ziel verfolgt, die folgenden Forschungsfragen zu beantworten:

1. Welches Szenario (mit Patentierung vs. ohne Patentierung) bietet den Akteuren aus individueller Sicht einen größeren Anreiz zu innovieren?

2. In welchem Szenario gibt es aus makroökonomischer Perspektive eine höhere Innovationsrate, d. h. mehr Perioden in denen innoviert wird?

3. Wie bedeutend sind die Monopolisierungstendenzen durch Patentierung?

Im Folgenden beschreiben wir zunächst den juristischen Hintergrund der Diskussion (Kapitel 2), bevor wir in Kapitel 3 ökonomische Aspekte zu Patenten, speziell Softwarepatenten diskutieren. In Kapitel 4 wird das ökonomische Modell und in Kapitel 5 erste simulative Ergebnisse dargestellt. Der Artikel schließt mit einer kurzen Zusammenfassung und einem Ausblick.

\section{Die Rechtslage in Deutschland}

Das Patentrecht ist in Deutschland Teil des gewerblichen Rechtsschutzes [Weis88]. Für die Frage der Patentierfähigkeit von Computerprogrammen ist $\S 1$ des Patentgesetzes (PatG) von 1981 bindend. Patente werden gemäß Absatz 1 für Erfindungen erteilt, die neu sind, auf einer erfinderischen Tätigkeit beruhen und gewerblich anwendbar sind. Bislang vom Patentrecht ausgeschlossen sind:

1. Entdeckungen sowie wissenschaftliche Theorien und mathematische Algorithmen,

2. ästhetische Formschöpfungen,

3. Pläne, Regeln, Verfahren für gedankliche Tätigkeiten, für Spiele oder für geschäftliche Tätigkeiten sowie Programme für Datenverarbeitungsanlagen und

4. die Wiedergabe von Informationen.

Sie stehen der Patentfähigkeit allerdings nur sofern entgegen, als für die genannten Gegenstände und Tätigkeiten ,als solche “ Schutz begehrt wird [Comp00, S. 150]. Allerdings fehlt hier jede Definition seitens des Gesetzgebers, sodass bspw. eine Abgrenzung in patentierfähige und nicht patentierfähige Softwareprogramme für Datenverarbeitungsanlagen äußerst schwer fällt.

Patentschutz ist in Deutschland auf maximal zwanzig Jahre begrenzt. Wesentlicher Bestandteil des Patentantrages ist eine detaillierte Beschreibung der Erfindung, die so ausführlich gehalten sein muss, dass ein Fachmann sie nacharbeiten kann. Sind die notwendigen Kriterien erfüllt, erteilt das Patentamt das Patent [BeKr86, S. 4]. 
Der Gegenstand darf nicht bereits bekannt sein (Neuheit) und darf sich auch nicht aus dem Bekannten (dem sog. Stand der Technik) für den Durchschnittsfachmann in naheliegender Weise ergeben (erfinderische Tätigkeit). Das Erfordernis der Neuheit stellt sicher, dass ein Patent niemals der Allgemeinheit etwas nehmen kann, was bereits der Öffentlichkeit zugänglich gemacht worden ist, also zum freien Wissen gehört [Comp00, S. 150]. Der Tatbestand der gewerblichen Anwendung gilt dann als gegeben, wenn die Erfindung auf irgendeinem gewerblichen Gebiet hergestellt oder eingesetzt werden kann [Comp00, S. 151].

Neben den genannten Kriterien ist Technizität die bedeutendste Vorrausetzung der Patentierfähigkeit von Gegenständen und Verfahren. In einem Beschluss des Bundesgerichtshofs (BGH) vom 27. März 1969 wurde definiert:

„(...)Dem Patentschutz zugänglich ist eine Lehre zum planmäßigen Handeln unter Einsatz beherrschbarer Naturkräfte zur Erreichung eines kausal übersehbaren Erfolges (...) [BGHG69].

Die grundsätzliche Aussage ist, dass nur ,technische“ Erfindungen dem Patentschutz zugänglich sind. Die Frage, ob Computerprogramme als solche zu dem Gebiet technischer Erfindungen zählen, ist nicht leicht zu beantworten. Der Bundesgerichtshof hat bspw. in den Entscheidungen „Sprachanalyseeinrichtung“, „Logikverifikation“ [BGHG00] oder „Merkmale zur Steuerung des Programms“ [BGHG86] die zugrunde liegende Technizität unbestritten bestätigt und auf diese Verfahren oder Methoden Patentschutz gewährt. In Fällen, in welchen es sich um betriebswirtschaftliche Software, bspw. Textverarbeitungsprogramme oder betriebswirtschaftliche Optimierungssoftware handelte, bestritt der BGH das Erfordernis der Technizität und lehnte Patentschutz ab [BGHG92; BGHG99].

In der Rechtsprechung existieren, bezogen auf die Fragestellung der Technizität, zwei gegensätzliche Ansätze: Die so genannte Kerntheorie und die Gesamtbetrachtungstheorie. Bei der Kerntheorie wird der Inhalt des Anspruchs mit dem bekannten Stand der Technik verglichen und die der Fachwelt vermittelte Lehre so auf den gegenüber dieser Lehre als „Überschuss" verbleibenden, dann hinsichtlich der Technizität allein zu beurteilenden Rest eingeschränkt [Tauch97, S. 6]. In der Gesamtbetrachtungstheorie werden die vorgeschlagenen Lösungsmittel, aber auch das zu lösende Problem und die nach der Anweisung zu erzielenden Effekte, hinsichtlich der benötigten Technizität geprüft [Elbi00, S. 3]. Das Prüfungsschema hat sich in den letzten Jahren von der Kerntheorie hin zur Gesamtbetrachtungstheorie entwickelt. Ende der 70er Jahre wurden 100\% und 2000 lediglich noch etwa $50 \%$ der Verfahren nach der Kerntheorie entschieden [Blind ${ }^{+} 01$, S. 138]. Dies ist sicherlich ein Grund, warum Computerprogrammen vermehrt Patentierfähigkeit zugesprochen wird [Blind ${ }^{+} 01$, S. 138]. Die nachfolgende Tabelle verdeutlicht die 
Anzahl der Patentanmeldungen der Jahre 1997-2000 in der Klasse Datenverarbeitung $^{l}$ in Deutschland.

\begin{tabular}{|c|c|c|c|c|}
\cline { 2 - 5 } \multicolumn{1}{c|}{} & \multicolumn{4}{c|}{ Jahr } \\
\cline { 2 - 5 } & 1997 & 1998 & 1999 & 2000 \\
\hline Angemeldete Patente & $\mathbf{1 4 3 8}$ & $\mathbf{1 4 2 9}$ & $\mathbf{1 9 2 1}$ & $\mathbf{1 5 6 6}$ \\
\hline
\end{tabular}

Tabelle 1: Anmeldungen der Klasse Datenverarbeitung beim DPMA [DPMA00, S. 18]

\section{3 Ökonomische Bedeutung von Patenten}

Aus mikroökonomischer Perspektive werden Patente häufig mit dem Schutz vor Imitation begründet. Patente werden dabei als Anreiz verstanden, Innovationskosten zu tragen, um diese einschließlich der Kosten für die Verwaltung durch positive Einnahmen zu kompensieren und zu sichern [BeRa91, S. 5]. Hierdurch wird dem Inhaber eine zeitlich begrenzte Monopolstellung verliehen.

Die Softwareentwicklung ist durch kurze Innovationszyklen, hohe Dynamik, und damit kurze Produktlebenszyklen bzw. -laufzeiten gekennzeichnet [Fried ${ }^{+} 02, \mathrm{~S}$. 155]. Der Patentschutz bietet diesbezüglich zwei wesentliche Vorteile:

- Der Patentinhaber kann durch seine Monopolstellung höhere Preise verlangen.

- Der Zeitraum, in dem der Inhaber Gewinne aus seiner Innovation ziehen kann, wird künstlich verlängert.

Darüber hinaus kann er durch die Vergabe von Lizenzen an zukünftigen Entwicklungen Dritter partizipieren [Ordo91, S. 50]. Bessen/Maskin sind trotz erfolgreicher Lizenzierungsbeispiele, etwa MP3 [Endr01, S. 20] allerdings der Ansicht, dass Lizenzierung Wettbewerb impliziert und somit die Monopolgewinne des Patentbesitzers gefährdet [BeMa00, S. 3]. Wenn überhaupt, würden Lizenzen zu Kosten, die sich knapp unterhalb des Marktwertes der Weiterentwicklung befinden, vergeben. Dies führt zwangsläufig dazu, dass Investitionen in F\&E ausbleiben (holdup problem) [Chan95, S. 35]. Diese Unterinvestitionen können auch dann entstehen, wenn ein Innovator Lizenzen vergibt, jedoch seine eigenen F\&EInvestionen reduziert [Chan95, S. 35].

$1 \quad$ Softwarepatente werden i.d.R. in der Klasse Datenverarbeitung angemeldet, allerdings läßt sich nicht ausschliessen, dass auch in anderen Klassen Patente auf Computerprogramme erteilt werden. 
Mit der Patentierung ist eine Offenlegung des Patentanspruches von 6 Monaten verbunden. Diese Offenlegung von Informationen erschwert insbesondere für klein- und mittelständische Unternehmen (KMU) die Patentierung von Entwicklungen. Über die Offenlegung können etwa Konkurrenten schon vor der Vermarktung des Produktes auf die eigentliche Erfindung aufmerksam gemacht werden, mit den Folgen von Imitationen und daraus resultierenden Rechtsstreitigkeiten. Zusätzlich bilden Patentierungskosten häufig eine unüberwindbare Barriere (die Kosten zur Beantragung eines europäischen Patents summieren sich auf min. $€$ 30.000 [Beck02, S. 1]). Auf der anderen Seite trägt die Offenlegungspflicht von Patenten aus makroökonomischer Sicht zu einer Verbreitung von Informationen bei, und hat eine positive Wirkung auf die Faktorproduktivität von Volkswirtschaften [ThRu99, S. 68].

Ein weiteres Spannungsfeld in der Patentierungsdebatte stellen Ressourcenfehlallokationen dar. Wenn eine Neuerung lediglich von einem begrenzten Nutzerkreis beansprucht wird, können Patente aufgrund ihrer Kosten aus ökonomischer Sicht eine sub-optimale Lösung implizieren. In diesem Kontext sind beispielsweise die Arbeiten von Klemperer sowie von Gilbert/Shapiro zu nennen [GiSh90; Klem90]. Werden neben den Kosten des Innovators, der den Zuschlag für ein Patent erhält (die Literatur spricht vom Gewinner des Wettlaufs) auch die Kosten derjenigen Unternehmen berücksichtigt, die den Wettlauf um die Patentierung nicht für sich entscheiden konnten, verstärkt sich dieses Argument [DaSt80, S. 267].

Jaffe vertritt die Meinung, dass Patente grundsätzlich die Innovationsaktivitäten Dritter blockieren, indem Lizenzen für Weiterentwicklungen verweigert werden. Die Innovationsdynamik einer Volkswirtschaft kann somit beeinträchtigt werden, er bezeichnet dies als negativen Nettoeffekt [Jaff99, S. 16]. Rivette/Kline bezeichnen diese Strategie als bracketing [RiK100, S. 31]. Um Blockaden zu entgehen, bauen Unternehmen häufig Patentportfolios auf, damit diese dann in Verhandlungen mit Konkurrenten eingesetzt werden können. Kash/Kingsten sprechen von Kreuzlizenzen als bargaining chips im Wettbewerb [KaKi01, S. 16]. Der Begriff der Kreuzlizensierung wird verwendet, wenn Unternehmen gegenseitig Nutzungsrechte zur Anwendung einer Innovation austauschen. Harhoff/Reitzig bezeichnen Patente gar als Waffe im Wettbewerb und als entscheidende Verhandlungsmasse in Lizenzierungsverfahren mit anderen Unternehmen [HaRe00, S. 9].

Betrachtet man den Softwaremarkt genauer, müssen Netzeffekte betrachtet werden. Diese beschreiben den Tatbestand, dass es zwischen dem Nutzen, den ein Gut stiftet, und der Anzahl der Anwender eine wechselseitige positive Abhängigkeit gibt [FaSa86, S. 940; KaSa86, S. 822]. Patente und Netzeffekte werden häufig im Spannungsfeld zwischen Produktvielfalt und Standardisierung diskutiert. Hierbei steht vor allem das Konzept der Kompatibilität im Mittelpunkt.

Märkte, die Netzeffekten unterliegen, neigen zu Monopolen, da die schnelle Verbreitung einer Technologie zu einem De-facto-Standard führt. Hierbei besteht nun die Gefahr, dass der Durchsetzung einer technisch überlegenen Innovation ein 
durch eine große installierte Basis verursachter Lock-in in eine bereits bestehende, minderwertige Technologie entgegensteht [FaSa86, S. 940f]. Standards können demnach in gewisser Weise Innovationen und somit Produktvielfalt behindern. Patente können diesen Effekt verstärken. Auf der anderen Seite erzeugen Standards Kompatibilität zwischen den die Standards erfüllenden Produkten, mit dem Effekt steigender Kombinationsmöglichkeiten von Produkten [Blind ${ }^{+}$01, S. 21]. Dies gelingt aber nur dann, wenn die notwendige Interoperabilität zwischen Systemen und Anwendungen bzw. zwischen verschiedenen Anwendungen gegeben ist. Ohne diese Interoperabilität fehlt vor allem KMU die Möglichkeit, auf große Kundennetzwerke zuzugreifen, da sie i.d.R. nicht in der Lage sind, Netzwerke auf Basis eigener Werksnormen aufzubauen [FaSa86, S. 254]. Das Fehlen offener Schnittstellen kann somit zu einem Lock-in von Altkunden und damit zu ,monopolartigen“ Netzwerkstrukturen führen, weil die Wechselkosten der jeweiligen Teilnehmer zu hoch sind [MeSz01, S. 31]. Patentierte (und nicht offene) Schnittstellen können dieses Phänomen verstärken.

In Deutschland wird in rund 19.200 Unternehmen - 10.568 der Primärbranche (DV-Dienstleister, Hersteller von Datenverarbeitungsgeräten und -einrichtungen) und 8.650 der Sekundärbranchen (Maschinenbau, Elektrotechnik, Fahrzeugbau, Telekommunikation und Finanzdienstleistungen) - Software entwickelt bzw. angepasst [GfKM00, S. 4].

Tabelle 2 zeigt die Beschäftigtenstruktur beider Branchen.

\begin{tabular}{|lr|r|}
\hline Beschäftigte & Primärbranche & Sekundärbranche \\
\hline 1 bis 9 & 8173 & 4058 \\
\hline 10 bis 49 & 1735 & 2242 \\
\hline 50 bis 199 & 475 & 1326 \\
\hline 200 und mehr & 185 & 1364 \\
\hline Gesamt & $\mathbf{1 0 5 6 8}$ & $\mathbf{8 9 9 0}$ \\
\hline
\end{tabular}

Tabelle 2: Struktur der Primär- und Sekundärbranche [GFKM00, S. 55]

Die genauere Untersuchung von Softwareentwicklungsprozessen verdeutlicht weitere Kriterien und Unterscheidungsmerkmale zu anderen Branchen.

\section{a. Sequenzialität und Komplementarität}

Sequenziell bedeutet, dass Erfindungen auf vorangegangenen aufbauen, so wie Windows auf DOS, und es eine hohe Frequenz an inkrementellen Weiterentwicklungen (Derivaten) gibt. Unter Komplementarität wird verstanden, dass jeder potentielle Innovator einen etwas anderen Entwicklungsansatz wählen kann, und dadurch die Gesamtwahrscheinlichkeit erhöht wird, ein bestimmtes Ziel innerhalb 
einer vorgegebenen Zeit zu erreichen [BeMa00, S. 11; Blind ${ }^{+} 01$, S. 65.]. Weitere Arbeiten hinsichtlich vorliegender Sequenzialität und Komplementarität wurden beispielsweise von Green/Scotchmer oder Chang durchgeführt [GrSc95; Chan95].

\section{b. Nutzung und Verfügbarkeit von „quelloffener Software“}

Der für Neu- und Weiterentwicklungen benötigte Input besteht mittlerweile aus bis zu 20\% quelloffener Software [Fried ${ }^{+} 02$, S. 158]. Unter ,quelloffener Software“ versteht man Programme, deren Quellcode (häufig kostenlos) Dritten zur Nutzung und Weiterentwicklung zur Verfügung gestellt wird.

\section{c. Digitaler Charakter von Softwareprodukten}

Die speziellen Eigenschaften von Software, insbesondere ihre digitale Struktur, ermöglichen eine quasi unbegrenzte Replizierbarkeit. Dies kann zu einer drastischen Verringerung der Gewinnphase des Entwicklers führen. Emery ist der Ansicht, dass deshalb Innovatoren Patentschutz auf Verfahren und Produkte erhalten sollten [Emer96, S. 30ff]. Entwicklungs- und letztlich auch Vermarktungszyklen von Software unterliegen einer anderen Zeitrechnung, einem normalen Jahr werden im Allgemeinen 4-7 Internetjahre zugerechnet [Karl02]. In diesem Zusammenhang werden insbesondere die langwierigen Erteilungsverfahren von Patenten kritisiert. Innovationen haben sich bis zur Erteilung eines Patentes häufig verändert, bzw. sind durch eigene oder von Konkurrenten hergestellte Weiterentwicklungen abgelöst worden [Jaff99, S. 3]. Die gesetzliche Regelung einer 20-jährigen Patentlaufzeit steht dann in keiner vernünftigen Relation zur Dynamik des Marktes. Darüber hinaus zeichnet sich der Softwaremarkt durch hohe Komplexität und Unübersichtlichkeit aus. Nicht immer ist der Beitrag eines bestimmten Entwicklers am Endprodukt genau zu definieren. Hinzu kommt, dass häufig an Entwicklungsstellen parallel gearbeitet werden kann, und der Stand der Technik selten vollständig überschaubar ist [KaKi01, S. 14]. Nicht zuletzt aufgrund dieser Widrigkeiten ist es für Softwareentwickler nahezu unmöglich Patentverletzungen zu identifizieren und ihre Folgen abzuschätzen [Smet01]. Tabelle 3 veranschaulicht Vor- und Nachteile von Softwarepatenten: 


\begin{tabular}{|l|l|}
\hline \multicolumn{1}{|c|}{ Positive Aspekte } & \multicolumn{1}{c|}{ Negative Aspekte } \\
\hline - Schutz vor Imitation der Innovation so- & - Zu den F\&E - Kosten fallen hohe Patentie- \\
wie Verringerung der Produktpiraterie. & rungskosten an. \\
- Verlängerung der „Monopolphase“ am & - Patentverletzungen sind nur schwer und \\
Markt (größere Gewinne). & äußerst teuer kontrollierbar. \\
- Patente verstärken den ,first mover ad- & - Die Entwicklungskosten werden durch \\
vantage“ und somit eine De-facto- & Lizenzzahlungen erheblich erhöht. \\
Standardisierung (positive Rückkopp- & - Die Offenlegungspflicht bei Patenten kann \\
lung). & zu Ideendiebstahl führen. \\
- Patente repräsentieren Aktiva, die bilan- & - Die Reichweite von Patenten kann kom- \\
ziert werden können, um bspw. die Ver- & plementäre Entwicklungswege verhindern. \\
handlungen mit Banken zu erleichtern. & - Die inkrementelle Weiterentwicklung wird \\
- Der Aufwand für Entwicklungskosten & durch Patente behindert. \\
wird durch die Möglichkeit, Monopolist & - Patente können notwendige Interoperabili- \\
zu sein und höhere Preise verlangen zu & tät behindern. \\
können, überkompensiert. & - Blockadestrategien und Monopole können \\
- Durch Lizenzierung können weitere Ge- & zu Unterversorgungen in F\&E führen. \\
winne erwirtschaftet werden. & - Durch Patente wird die Produktvielfalt \\
- Die Offenlegungspflicht verbreitet den & erschwert. \\
Wissensfluss. & - Patente können zu Fehlallokationen führen \\
& (Mehr Rechtspflege als F\&E). \\
\hline
\end{tabular}

Tabelle 3: Vor- und Nachteile von Patenten auf Software

\section{Ein Simulationsmodell}

\subsection{Annahmen}

Die folgenden Annahmen basieren auf der vorangegangenen deskriptiven Analyse der ökonomischen Literatur sowie der Bestandsaufnahme der juristischen Situation in Deutschland.

1. Der Softwaremarkt ist durch kurze Innovationszyklen gekennzeichnet. Derivative Verbesserungen entstehen in weniger als 12 Monaten.

2. Der Softwaremarkt ist durch ein hohes Maß an inkrementellen derivativen Weiterentwicklungen basistechnischer Innovationen gekennzeichnet. Der Markt wird als in hohem Maße sequenziell bezeichnet. 
3. Der Markt für Software besitzt einen hohen Grad an Komplementarität.

4. F\&E-Aufwendungen veralten nach ca. 3-4 Perioden.

5. Der Softwaremarkt ist durch einen hohen Anteil an KMU gekennzeichnet.

6. Der Softwaremarkt ist durch starke Netzeffekte gekennzeichnet.

7. Je größer die Aufwendungen in F\&E sind, desto größer ist die Erfolgswahrscheinlichkeit eine Innovation zu finden.

\subsection{Einflussfaktoren}

Auf Basis der unter 4.1 dargestellten Annahmen wurde ein ökonomisches Wahrscheinlichkeitsmodell entwickelt, welches einen unregulierten Softwaremarkt mit einem Markt vergleicht, der über die Möglichkeit Innovationen patentieren zu lassen verfügt. Die wesentlichen Ziele des Modells sind auf der einen Seite, das Innovationsverhalten im Zeitablauf zu vergleichen und auf der andern Seite den Grad der Monopolbildung zu messen.

\subsubsection{Strukturelle Faktoren}

Das Modell umfasst I Akteure, welche Softwareproduzenten in einem speziellen Segment, z. B. der Textverarbeitung, darstellen. Die Akteure konkurrieren in jeder Periode um Weiterentwicklungen in ihrem Segment. Pro Periode ist genau eine Weiterentwicklung möglich. Jeder Akteur kennt das gesamte Netzwerk und den aus einer Weiterentwicklung resultierenden ökonomischen Gewinn. Er kann folglich einen Erwartungswert über seinen Erfolg bilden.

Basierend auf den topologischen Daten des deutschen Softwaremarktes entfallen $80 \%$ des Netzwerkes auf die Klasse der Kleinunternehmen, $15 \%$ werden der Klasse der mittelständischen Unternehmen und 5\% den Großunternehmen zugeordnet. Der Planungshorizont beträgt $T$ Perioden.

Wir definieren, dass alle Akteure in jeder Periode $t(t=1 . . T)$ neu über ein mögliches Investitionsvorhaben in F\&E entscheiden müssen. Nur wenn diese Entscheidung positiv ausfällt und die Kosten aufgewendet werden, besteht die Möglichkeit eine Innovation zu finden.

Wird auf diese Innovation ein Patent erteilt, so läuft der so gewährte Imitationsschutz über $T_{p}$ Perioden.

\subsubsection{Monetäre Faktoren}

In jeder Periode führen eine oder mehrere erfolgreiche Innovationen zum ökonomischen Gewinn $v_{t}$. Dieser Gewinn wird im Modell ohne Patente unter den erfolgreichen Akteuren zu gleichen Teilen aufgeteilt. Ist Patentierung möglich, ent- 
fällt der Gewinn auf den Gewinner des Patentrennens (analog zum Modell von Bessen/Maskin (2000)).

$$
v_{t}=\sum_{i=1}^{I} v_{i t}=\bar{v} \quad \forall t=1 . . T
$$

Eine weitere Größe, die den erwarteten Profit beeinflusst, sind die F\&E - Aufwendungen $c_{i t}^{d}$ in jeder Periode. Je größer diese ausfallen, desto geringer fällt der erwartete Gewinn aus. Auf der anderen Seite beeinflusst die Höhe der Investition in F\&E jedoch die Erfolgswahrscheinlichkeit, überhaupt eine Innovation zu finden.

Wenn Patentierung möglich ist, dann müssen die Unternehmen, die investiert haben, und am Patentierungsprozess teilnehmen, zusätzlich zu den F\&E - Kosten, Kosten für der gesamten Patentierungsprozess $c_{i t}^{p}$ investieren:

I. Kosten der Erstellung der Patentschrift unter Zuhilfenahme von Patentanwälten,

II. Patentanmelde- und Erteilungskosten und

III. Kosten der Aufrechterhaltung von Patenten.

Die unter II und III aufgeführten Kosten müssen nur vom tatsächlichen Patentempfänger getragen werden, sind jedoch von untergeordneter Bedeutung.

\subsubsection{Wahrscheinlichkeitsparameter}

Die Erfolgswahrscheinlichkeit $p_{i}$ für eine Innovation in jeder Periode $t$ stellt den zentralen Parameter des Modells dar. Folgende Parameter beeinflussen die Wahrscheinlichkeit:

- F\&E-Kosten, welche in $t$ und den drei vorrangegangenen Perioden aufgewandt wurden: $c_{i t}^{d}, c_{i t-1}^{d}, c_{i t-2}^{d}, c_{i t-3}^{d}$ (Annahme 4).

- Innovationserfolge der vergangenen drei Perioden. Erfolgreiche Innovationen vergangener Perioden werden durch die Binärvariable $x_{i t-1}, x_{i t-2}, x_{i t-3}$ formal dargestellt. Diese ist eins, wenn Akteur $i$ eine Innovation in $t$ gefunden hat.

- Existenz von Patenten (ohne Lizenzierung): Derivative Verbesserungen einer patentierten Innovation durch andere in zukünftigen Perioden müssen auf einem, je nach Breite des Patentschutzes mehr oder weniger stark komplementären Weg vorgenommen werden (bestehender Code darf nicht verwendet werden). Hierdurch verringert sich die Erfolgswahrscheinlichkeit der Akteure, die nicht Inhaber des Patentes sind (Annahme 3). Dieser Effekt wird durch die Variable $\varepsilon_{i}$ repräsentiert. $\varepsilon_{i}$ verringert die Innovationswahrscheinlichkeit von $i$, wenn die Innovation in Periode $t$ von Akteur $j \neq i$ patentiert wurde. 


$$
\varepsilon_{i t}=\left\{\begin{array}{cl}
\varepsilon<1 & \text { if } z_{i t}=0 \wedge \sum_{i=1}^{I} z_{j t}=1 \\
1 & \text { else }
\end{array}\right.
$$

Die Binärvariable $z_{i t}$ reflektiert den erfolgreichen Patentierungsprozess von Akteur $i$ 's Innovation in Periode $t$.

Die Laufzeit von Patenten liegt derzeit bei 20 Jahren $\left(T_{p}=20\right)$, sodass der Einfluss eines Patents auf andere derivative Entwicklungen mit abnehmender Stärke 20 Jahre anhält.

Der Grad des Einflusses der aufgezeigten Parameter nimmt im Zeitablauf ab. Dieser Effekt wird durch die Faktoren $w_{d}$ und $w_{x}\left(0<w_{d}, w_{x}<1\right)$ für die jeweiligen Parameter $c_{i t}^{d}, c_{i t-1}^{d}, c_{i t-2}^{d}, c_{i t-3}^{d}$ und $x_{i t-1}, x_{i t-2}, x_{i t-3}$ realisiert (Formel 3).

$$
\mathrm{A}: \sum_{\tau=t-3}^{t} c_{i \tau}^{d} \cdot w_{d}^{(t-\tau)} ; \mathrm{B}: \sum_{\tau=t-3}^{t-1} x_{i \tau} \cdot w_{x}^{(t-\tau)} ; \mathrm{C}: \prod_{\tau=t-T_{P}}^{t-1} \varepsilon_{i \tau}^{\left(\tau-t+T_{P}+1\right)}
$$

Die Ausdrücke A und B werden auf die Basis 1 normalisiert. Die normalisierte Funktion wird mit einem „Brancheninnovationsmaß“ $M$ gewichtet. Je niedriger $M$ ist, desto leichter lassen sich Innovationen in einer Branche realisieren.

$$
\begin{gathered}
\alpha \cdot \frac{\sum_{\tau=t-3}^{t} c_{i \tau}^{d} \cdot w_{d}^{(t-\tau)}}{\sum_{\tau=t-3}^{t} \bar{c}_{i \tau}^{d} \cdot w_{d}^{(t-\tau)}}+\beta \cdot \frac{\sum_{\tau=t-3}^{t-1} x_{i \tau} \cdot w_{x}^{(t-\tau)}}{\sum_{\tau=t-3}^{t-1} w_{x}^{(t-\tau)}} \\
p_{i t}=\frac{\prod_{\tau=t-T_{P}}^{t-1} \varepsilon_{i \tau}^{\left(\tau-t+T_{P}+1\right)}}{M} \\
\operatorname{mit}_{i t}^{d} \quad \alpha, \beta \geq 0 ; \quad \alpha+\beta=1 \text { und } \quad M \geq 1 \\
c_{i t}^{d}=0, \quad \varepsilon_{i \tau}=1 \quad \forall t<1
\end{gathered}
$$

\subsubsection{Entscheidungsprozess}

Der Innovations- und Patentierungsprozess besteht aus 4 Prozessschritten in jeder Periode:

IV. Akteur $i$ muss eine Entscheidung treffen, ob er am Innovationsprozess teilnimmt und Entwicklungskosten von $c_{i t}^{d}$ investiert.

V. Der Innovationsprozess kann in Abhängigkeit der Erfolgswahrscheinlichkeit $p_{i t}$ erfolgreich $\left(x_{i t}=1\right)$ oder negativ verlaufen $\left(x_{i t}=0\right)$. 
VI. Wenn $x_{i t}=1$ ist, dann muss der Akteur entscheiden, ob er am Patentierungswettlauf teilnimmt $\left(y_{i t}=1\right)$ oder nicht $\left(y_{i t}=0\right)$.

VII. Von allen Akteuren, die am Wettlauf teilnehmen, kann nur einer das Rennen gewinnen. Der Gewinner wird mittels eines Zufallsgenerators mit gleicher Wahrscheinlichkeit für alle Akteure, die am Wettlauf teilnehmen, ermittelt. Wenn Akteur $i$ das Patent erwirbt, folgt $\left(z_{i t}=1\right)$, andernfalls $z_{i t}=0$.

Die Aktivitäten III und IV treten nur ein, wenn Patentierung möglich ist.

Ein risikoneutraler Akteur $i$ wird in $t$ nur in $\mathrm{F} \& \mathrm{D}$ investieren, wenn sein erwarteter Nutzen $E\left[v_{i t}^{\text {net }}\right]$ (Formel 5 - links) positiv ist (Schritt I). Der Erwartungswert hängt neben der individuellen Erfolgswahrscheinlichkeit für eine Innovation von den aktuell geleisteten Aufwendungen und der erwarteten Zahl der Innovatoren, mit denen der ökonomische Bruttogewinn geteilt werden muss, ab. Im Patentierungsfall reduziert der Patentbesitzer über $\mathcal{E}$ die Erfolgswahrscheinlichkeiten der anderen Akteure (Formel 6), sodass er von einer geringeren erwarteten Zahl von Innovatoren ausgehen kann, mit denen er $v_{t}$ teilen muss.

$$
E\left[v_{i t}^{\text {net }}\right]=\frac{v_{t}}{E\left[\sum_{i=1}^{I} x_{i t}\right]} \cdot p_{i t}-c_{i t}^{d} ; \quad u_{i t}=\left\{\begin{array}{ll}
1 & \text { wenn } E\left[v_{i t}^{\text {net }}\right]>0 \\
0 & \text { sonst }
\end{array}\right\}
$$

Als Schätzer für die erwartete Anzahl an gefundenen Innovationen $E\left[\sum_{i=1}^{I} x_{i t}\right]$, wird die Anzahl der Akteure herangezogen, die in der Vorperiode innoviert haben.

$$
E\left[\sum_{i=1}^{I} x_{i t}\right]=\left\{\begin{array}{lll}
\sum_{i=1}^{I} x_{i t-1} \cdot \varepsilon^{T p} & \text { falls } z_{i t-1}=1 \\
\sum_{i=1}^{I} x_{i t-1} & \text { sonst } & \text { u.d.B. } E\left[\sum_{i=1}^{I} x_{i t}\right] \geq 1
\end{array}\right.
$$

Im Fall der Möglichkeit der Patentierung, sieht das Modell eine weitere Entscheidungsfunktion (Schritt III) vor. Der Entscheidungsträger muss sich nach Auffinden der Innovation entscheiden, ob er am Patentrennen teilnimmt, oder nicht.

Die Entscheidungsfunktion berücksichtigt die Erfolgswahrscheinlichkeit, das Patent zu erhalten, die deterministisch anfallenden Kosten für die Patentschrift sowie den Barwert der zukünftigen erwarteten Mehreinnahmen durch die für die anderen Entwickler aufgerichtete Marktbarriere (Formel 7). Die Binärvariable $y_{i t}$ repräsentiert die resultierende Entscheidung, am Patentwettlauf teilzunehmen. 


$$
y_{i t}=\left\{\begin{array}{ccc}
1 & \text { if } & \frac{v_{t}}{I}-c^{p}+E\left[B W_{t}^{P Z}\right] \geq 0 \\
& \sum_{i=1}^{I} x_{i t} \\
0 & \text { else }
\end{array}\right.
$$

Die Barwertfunktion zur Ermittlung der erwarteten Mehreinnahmen während der Patentlaufzeit ( $T_{p}$ Perioden) ist in Formel 8 dargestellt und wird im Appendix hergeleitet.

$$
E\left[B W_{t}^{P Z}\right]=\sum_{\tau=1}^{T_{p}}\left(v_{t+\tau} \cdot p_{i t} \cdot \frac{\left(1-\varepsilon^{T_{P}-\tau+1}\right)}{I \cdot \hat{p}_{t+\tau}^{o p} \cdot \varepsilon^{T_{P}-\tau+1}} \cdot q^{\tau}\right)
$$

$\hat{p}_{t+\tau}^{o p}$ ist der Schätzer für die in Periode $\mathrm{t}+\tau$ im Markt vorhandene durchschnittliche Innovationswahrscheinlichkeit, falls der Akteur nicht patentiert, und $q$ stellt den Diskontierungsfaktor dar. 


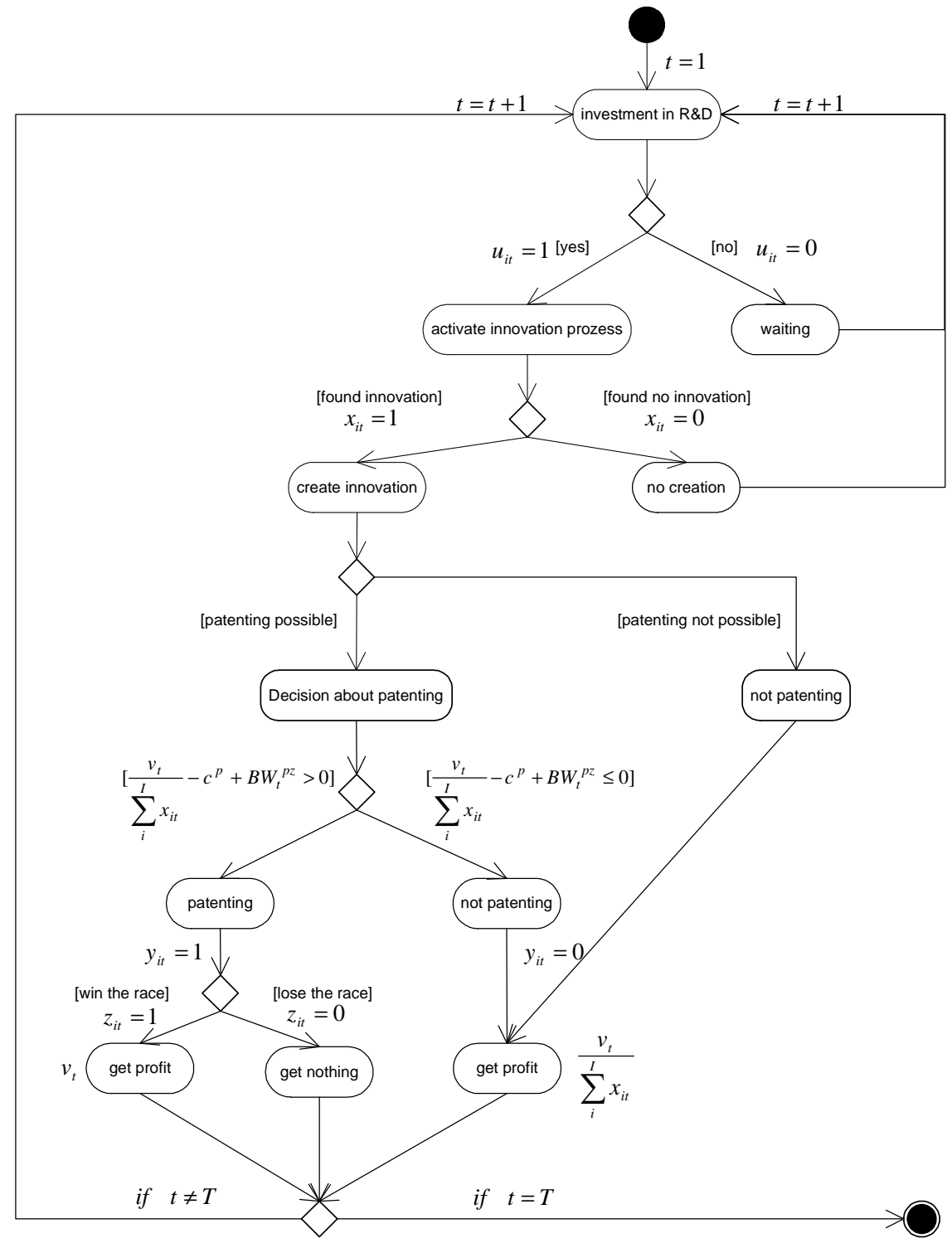

Abbildung 1: Aktivitätsdiagramm

Aus makroökonomischer Perspektive besteht nun das Hauptziel darin, die Innovationsrate zu maximieren, d. h. zu erreichen, dass in jeder Periode die Innovation durch min. einen der Akteure gefunden wird. 


$$
\max \sum_{t=1}^{T} x_{t} \text { mit } x_{i}=\max \left(x_{i t}, \ldots . x_{I t}\right)
$$

Nachfolgend werden nochmals die Forschungsfragen und Wege zur Beantwortung kurz dargestellt:

1. Welches Szenario (mit Patentierung vs. ohne Patentierung) bietet den Akteuren aus individueller Sicht einen größeren Anreiz zu innovieren?

2. In welchem Szenario gibt es aus makroökonomischer Perspektive eine höhere Innovationsrate, d.h. mehr Perioden in denen innoviert wird?

3. Wie bedeutend sind die Monopolisierungstendenzen durch Patentierung?

Frage 1 wird durch die kumulierte Differenz der Erwartungswerte in beiden Szenarien beantwortet: $\Delta \sum E[v]=\sum_{t=1}^{T} E^{o p}\left[v_{i t}^{n e t}\right]-E^{p}\left[v_{i t}^{n e t}\right]$. Positive Ergebnisse beschreiben den Tatbestand, dass die Anreize im Szenario ohne Patentschutz höher sind und umgekehrt.

Die zweite Frage wird durch Formel 14 beantwortet, und ebenfalls als Differenz aus beiden Szenarien dargestellt.

Zur Beantwortung der dritten Frage wird der Herfindahl-Index herangezogen. Häufig wird er als Marktkonzentrationsmaß verwendet. Im vorliegenden Modell wird er zunächst auf herkömmliche Weise in jeder Periode ermittelt. Um die Stabilität von Monopolen in der Zeit zu messen, wird weiterhin ein intertemporaler Herfindahl-Koeffizient $H K I$ ermittelt, der die Zahl der temporären Monopole eines Akteurs im Verhältnis zur Gesamtzahl aller Monopole innerhalb der simulierten Perioden als Marktanteile betrachtet.

$$
H K_{t}=\sum_{i=1}^{I}\left(\frac{v_{i t}{ }^{2}}{v_{t}} \quad H K I=\sum_{i=1}^{I}\left(\frac{\sum_{t=1}^{T} z_{i t}}{\sum_{i=1}^{I} \sum_{t=1}^{T} z_{i t}}\right)^{2}\right.
$$




\section{Erste Simulationsergebnisse}

\subsection{Parametrisierung}

Die Simulationen wurden mit einer kleinen Basis von 20 Akteuren unterschiedlicher Größe (Softwareanbieter) und T=50 durchgeführt. Die Größenstruktur entspricht der des deutschen Softwaremarktes, wobei die ersten beiden Kategorien zusammengefasst worden sind (Tabelle 2). Die Forschungs- und Entwicklungskosten sind gleichverteilt und der totale ökonomische Profit beträgt konstant 100 Geldeinheiten pro Periode. Die drei Unternehmensklassen unterscheiden sich in der Höhe ihrer Entwicklungskosten, deren jew. Bandbreiten in Tabelle 4 angegeben sind. Die Patentierungskosten sind aufgrund der kleinen Zahl von 20 Akteuren vernachlässigbar, da sie eine unrealistische Höhe annehmen müssten, um entscheidungsrelevant zu sein.

\begin{tabular}{|l|l|l|l|}
\hline Unternehmensgröße: & Groß & Mittel & Klein \\
\hline$c_{i}^{d}$ Obergrenze & 5 & 3 & 1 \\
\hline$c_{i}^{d}$ Untergrenze & 2 & 0,5 & 0 \\
\hline
\end{tabular}

Tabelle 4: Verteilung der Entwicklungskosten

In Tabelle 5 sind die übrigen Parameter, die zur Bildung der individuellen Innovationswahrscheinlichkeiten notwendig sind, angegeben.

\begin{tabular}{|l|c|c|}
\hline \multicolumn{3}{|c|}{ Wahrscheinlichkeitsparameter } \\
\hline Gewichtung früherer Entwicklungskosten & $w_{d}$ & 0,9 \\
\hline Gewichtung früherer erfolgreicher Innovationen & $w_{x}$ & 0,9 \\
\hline Relative Gewichtung der Entwicklungskosten & $\alpha$ & 0,5 \\
\hline Relative Gewichtung der erfolgreichen Innovationen & $\beta$ & 0,5 \\
\hline Gewichtung der Marktinnovationsschwierigkeit & $M$ & 1,0 \\
\hline
\end{tabular}

Tabelle 5: Konstante Wahrscheinlichkeitsparameter

Im Simulationsablauf wurde der Parameter der Patentschutzweite $\varepsilon$ von 1,0 ausgehend schrittweise um 0,001 reduziert (bis 0,8), wobei jede Konstellation 100mal simuliert wurde. 


\subsection{Ergebnisse}

Aufgrund der gewählten Parameter und der kleinen Branchengröße von 20 Akteuren sind die folgenden Darstellungen in ihrer Aussagekraft zunächst auf die simulierte Konstellation beschränkt. Ziel weiterer Arbeiten wird es sein, die Stabilität der Ergebnisse in großen Netzen mit realistischen Kostenstrukturen zu prüfen und die Sensitivitäten der einzelnen Faktoren zu analysieren.

\subsubsection{Anreizwirkung von Patenten aus individueller Sicht}

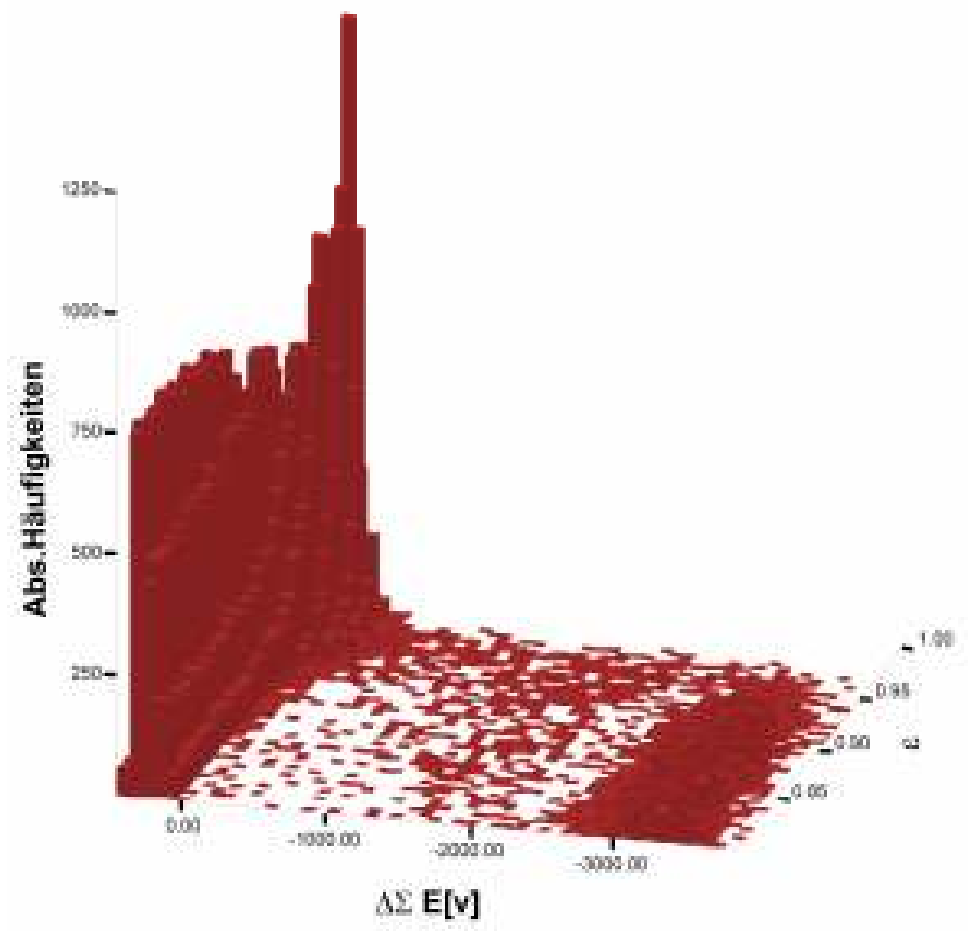

Abbildung 2: Kumulierte Differenz der Erwartungswerte $E^{o p}\left\lfloor v_{i t}^{\text {net }}\right]-E^{p}\left\lfloor v_{i t}^{\text {net }}\right\rfloor$

Abbildung 2 vergleicht die kumulierte Anreizwirkung des Szenarios ohne Patentierungsmöglichkeit (op-Szenario) mit der „Welt“ möglicher Patentierungsoption (p-Szenario). In der Ebene sind unterschiedliche kumulierte Erwartungswertdifferenzen bei Variation des Patentweitenparameters $\varepsilon$ abgetragen. Das Histogramm zeigt die Verteilung der Akteure aus 2000 Simulationsläufen gemäß ihrer individuellen Anreize. Die ,höchste“ Säule findet sich für $\varepsilon=1,0$ und $\Delta \Sigma E[v]=0,0$. Beide Szenarien verhalten sich auf diesem $\varepsilon$-Level identisch, da ein 
Patent keine Wirkung auf das Innovationsverhalten der Entwickler hat. Bei Reduktion von $\varepsilon$ bewegt sich die Differenz der Erwartungswerte für den überwältigenden Teil der Akteure in den positiven Bereich. Die Ausreißer, die stark negative Innovationsanreizdifferenzen erwarten, repräsentieren erfolgreiche $\mathrm{Pa}-$ tentinhaber im p-Szenario, die natürlich verglichen mit dem op-Szenario deutlich höhere Anreize haben, nachdem sie ihre Alleinstellung im Markt etabliert haben. Aus diesem Zusammenhang lässt sich ableiten, dass unter der gegebenen Parameterkonstellation aus mikroökonomischer Perspektive Patente im Softwaremarkt nicht zu verstärkter Anreizwirkung führen.

\subsubsection{Anreizwirkung von Patenten aus makroökonomischer Sicht}

In der nächsten Abbildung ist dargestellt, wie groß die Differenz der gefundenen Innovationen (Gleichung 14) zwischen den beiden Szenarien ist. Die Ordinate gibt an, wie stark das op-Szenario das p-Szenario dominiert. Dabei ist die Streuung durch die randomisierten Teile des Simulationsmodells erwartungsgemäß hoch. Auch hier ist bei $\varepsilon=1,0$ die Differenz gleich Null, da die Szenarien hier identisch sind. Interessant ist der etwa bei 0,95 liegende Patentierungs-Gap (am wenigsten gefundene Innovationen).

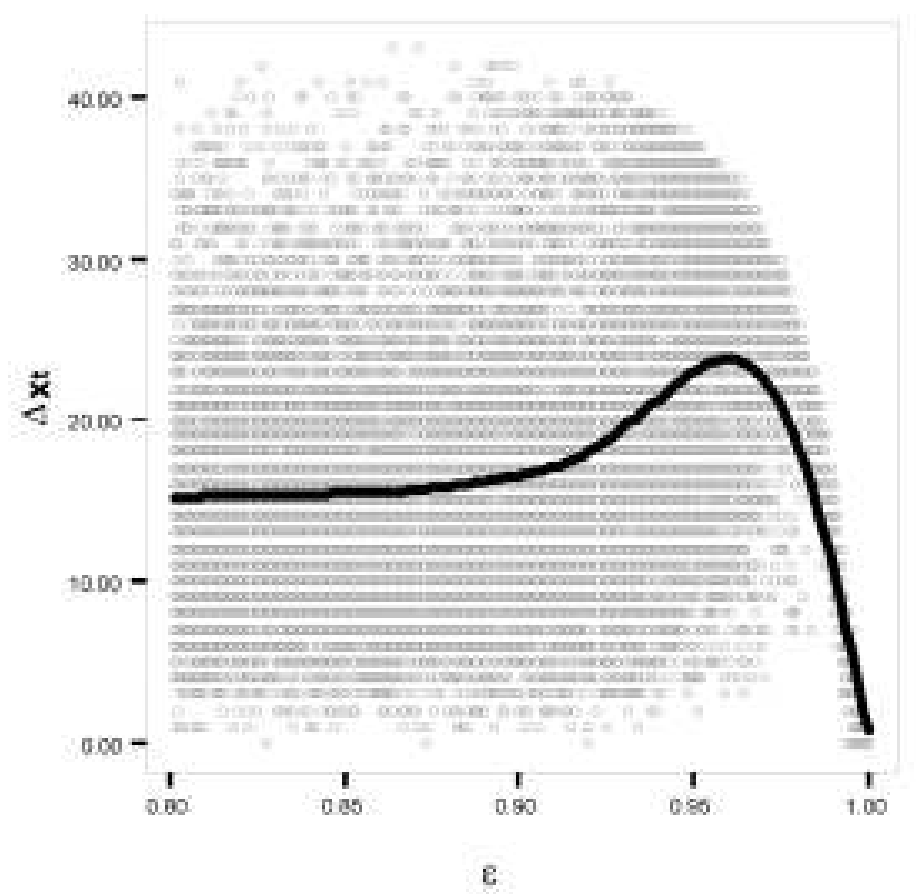

Abbildung 3: Differenz der gefundenen Innovationen: $\Delta \Sigma x_{t}$ in Abhängigkeit von $\varepsilon$ 


\subsubsection{Monopolisierungstendenzen}

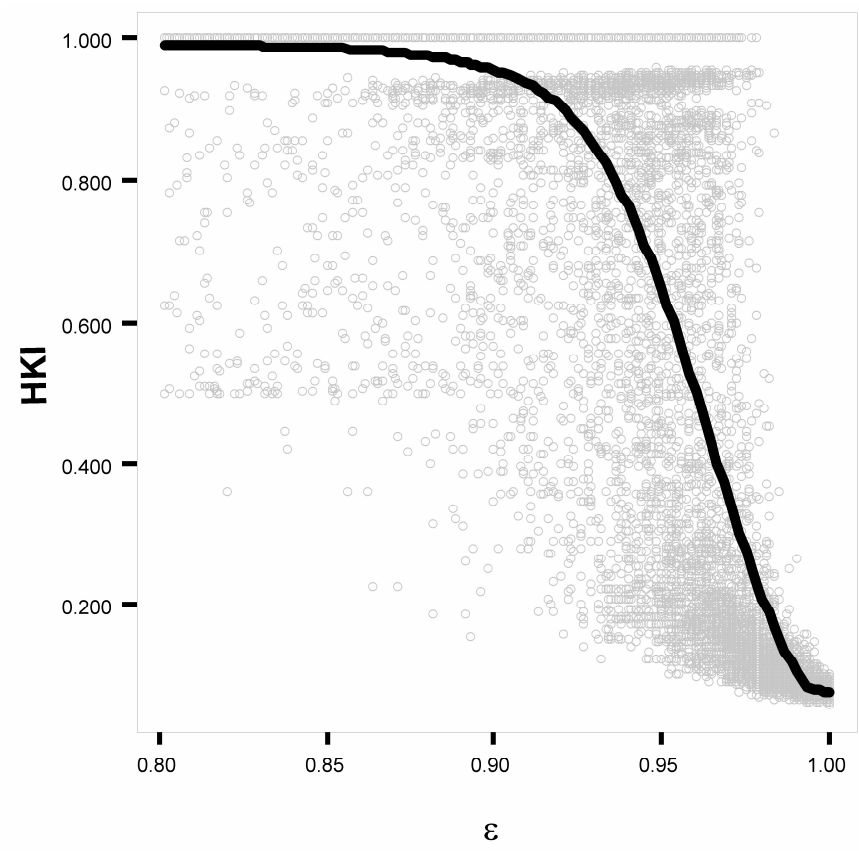

Abbildung 4: Marktkonzentration in Abhängigkeit der Schutzweite des Patents

Zur Beantwortung der dritten Frage wird der intertemporale HerfindahlKoeffizient $H K I$ herangezogen, der die Verteilung der Monopole über die unterschiedlichen erfolgreichen Innovatoren und Patentinhaber in der Zeit misst. Abbildung 3 zeigt, wie sich die Marktsituation mit wachsender Schutzweite von Patenten verändert. Wenn $\varepsilon=1,0$ ist, Patente also keine Wirkung haben, befindet sich der Markt in einem Polypol mit einer Konzentrationsrate nahe 0,05. Mit sich erweiterndem Patentschutz findet eine steigende Konzentration statt - bis zum völligen intertemporalen Monopol in der großen Mehrzahl der Fälle. Interessant ist jedoch auch bei niedrigen $\varepsilon$ eine leichte Häufung der Ergebnisse auf dem ,DuopolNiveau“ $(H K I=0,5)$. Dieses tritt dann ein, wenn ein Patentinhaber über eine längere Zeit keine neuen Innovationen findet und deswegen keine weiteren Patente anmelden kann. So steigen die Chancen der Wettbewerber, wieder in den Markt einzudringen und diesen mit einem eigenen Patent zu übernehmen. Über alle Perioden betrachtet finden in einem solchen Szenario wenige Innovationen statt, die recht gleichmäßig auf zwei Akteure aufgeteilt sind. 


\section{Zusammenfassung und Ausblick}

Gegenstand dieses Artikels sind Softwarepatente und ihr Einfluss auf die Innovationsaktivitäten innerhalb des Softwaremarktes. Besondere marktliche Bedingungen, wie sie im Softwaremarkt vorherrschen, lassen stärkere Zweifel an der grundsätzlichen Vorteilhaftigkeit von Softwarepatenten erkennen. Zum einen sind aufgrund hoher Sequenzialität Blockadestrategien deutlich wirkungsvoller als in anderen Märken und verstärken die aufgrund von Netzeffekten vorherrschenden Monopolisierungstendenzen. Zum anderen werden kompatible Entwicklungspfade bei umfassendem Patentschutz (niedriges $\varepsilon$ ) extrem erschwert (Kompatibilität), sodass die makroökonomische Gesamtwahrscheinlichkeit zum Finden einer Innovation reduziert wird. Die Simulationsergebnisse zeigen, dass im Softwaremarkt bereits die mikroökonomische (individuelle) Vorteilhaftigkeit von Patenten bezweifelt werden muss; aus makroökonomischer Sichtweise verstärkt sich dieses Ergebnis. Mit wachsender Schutzweite konzentriert sich der Markt bis hin zu einem langfristig stabilen Monopol.

Unsere weiteren Bemühungen richten sich zunächst darauf, die Simulationen mit einer größeren Basis durchzuführen, um die nicht unwichtigen Patentierungskosten berücksichtigen zu können. Die Simulationsergebnisse müssen noch stärker als bislang geschehen mit empirischen Daten werden. Zusätzlich soll die vieldiskutierte Laufzeit von Patenten variiert werden, sodass der Trade-off zwischen Anreiz durch verlängerte Monopolzeit und Blockade des Marktes differenzierter betrachtet werden kann. Außerdem ist geplant die Möglichkeit der Lizenzierung patentierter Innovationen in das Modell einzubauen, durch die die individuelle Anreizwirkung verstärkt wird.

\section{Literatur}

[Beck02, S.1] Beckmann, J.: Merkblatt Auslandspatente, 2002, S. 1-2. http://www.bepatent.de/MBpatwo.PDF, Abruf am 2003-02-10

[BeKr86] Bernhardt, W.; Krasser, R.: Lehrbuch des Patentrechts, Beck'sche Verlagsbuchhandlung, München, 1986.

[BeMa00] Bessen, J.; Maskin E.: Sequential Innovation, Patents, and Imitation, MIT Working Paper, Nr. 1, Cambridge, 2000.

[BeRa91] Besen, S. M.; Raskind, L. J.: An Introduction to the Law and Economics of Intellectual Property, in: Journal of Economic Perspectives Vol. 5, Nr. 1, 1991, S. 3-27.

[BGHG69] BGH GRUR 1969, 672, 675 - Rote Taube.

[BGHG86] BGH GRUR 1986, 307 - Digitale Signalverarbeitungsanordnung.

[BGHG92] BGH GRUR 1992, 36 - Chinesische Schriftzeichen. 
[BGHG99] BGH GRUR 1999, 1078 - Automaische Absatzsteuerung.

[BGHG00] BGH GRUR 2000, 498 - Logikverifikation; 930, 932 - Sprachanalyseeinrichtung.

[Blind $\left.{ }^{+} 01\right]$ Blind, K.; Edler, J.; Nack, R.; Straus, J.: Mikro- und makroökonomische Implikationen der Patentierbarkeit von Softwareinnovationen: Geistige Eigentumsrechte in der Informationstechnologie im Spannungsfeld von Wettbewerb und Innovation, im Auftrag des BmbF, Auftrag (36/00), 2000, S. 1-248.

[Chan95] Chang, H.: "Patent scope, antitrust policy and cumulative innovation," in: RAND Journal of Economics, Vol. 26, Nr. 1, 1995, S. 1-34.

[Comp00] Computerrecht, e-commerce, 4. Auflage, Beck-Texte im dtv, 2000.

[DaSt80] Dasgupta, P.; Stiglitz, J. E.: Industrial Structure and the Nature of Innovative Activity, in: Economic Journal Vol. 90, 1980, S. 266-293.

[DPMA00] Deutsches Patent- und Markenamt, Jahresbericht 2000, http://www.dpma.de/veroeffentlichungen/jahresberichte.html, Abruf am 2003-01-15.

[DPMA02] Deutsches Patent- und Markenamt, Pressemitteilung vom 12.03.2002, http://www.dpma.de/infos/pressedienst/pm020312a.html, Abruf am 2003-01-15.

[Endr01] Endres, A.: Wem nützen und wem schaden Software-Patente. In: Informatik Sektrum 24 (2001) 1, S. 19-24.

[Elbi00] Ebinger, T.: Bericht von der IT - Anwaltskonferenz 3 - Patentierbarkeit von Software, 17./18. November 2000, München, http://www.davit.de/langfassung\%20Bericht\%20Konferenz\%20Softwarepatente.rtf, Abruf am 2002-07-11.

[Emer96] Emery, S.: Innovation and Intellectual Property Protection: The Software Industry Perspective, in: The Columbia Journal of World Business Vol. 31, Nr. 1, 1996, S. 30-37.

[FaSa86] Farrell, J.; Saloner, G.: Installed Base and Compatibility: Innovation, Product Preannouncements, and Predation, in: The American Economic Review, Vol. 76, Nr. 5, 1986, S. 940-955.

[Fried $\left.{ }^{+} 02\right]$ Friedewald, M.; Blind, K..; Edler, J.: Die Innovationstätigkeit der deutschen Softwareindustrie, in WIRTSCHAFTSINFORMATIK, 44. Jahrgang, Heft 2, 2002, S. 151-161.

[GiSh90] Gilbert, R.; Shapiro, C.: Optimal Patent Length and Breadth, in: Rand Journal of Economics, Vol. 21, S. 106-112.

[GfKM00] GfK Marktforschung GmbH/Fraunhofer-Institut: Analyse und Evaluation der Softwareentwicklung in Deutschland, 2000, S. 1-249.

[GrSc95] Green, J.; Scotchmer, S.: "On the Division of Profit in Sequential Innovation", in: The RAND Journal of Economics, Vol. 26, 1995, S. 20-33.

[HaRe00] Harhoff, D.; Reitzig, M.: "Strategien zur Gewinnmaximierung bei der Anmeldung von Patenten", Arbeitsbericht, München, 2000, S. 1-24. 
[Jaff99] Jaffe, A. B.: The U.S. Patent System in Transition: Policy Innovation and the Innovation Process, National Bureau of Economic Research, Working Paper 7280, Cambridge, MA, 1999, S. 1-60.

[KaKi01] Kash, D. E.; Kingston, W. (2001): Patents in a world of complex Technologies, in: Science and Public Policy, Vol. 28, Nr. 1, 2001, S. 11-22.

[Karl02] Karle, R.; Die neuen Golddigger - Das WEB ist die Goldgrube des neuen Jahrtausends, http://www.unicum.de/beruf/b-03-00/ek7-0300.htm, Abruf am 2003-01-18.

[KaSh86] Katz, M. L.; Shapiro, C.: Technology adoption in the presence of network externalities, in: Journal of Political Economy, Vol. 94, Nr. 4, 1986, S. 822-841.

[Klem90] Klemperer, P.: How Broad Should the Scope of Patent Protection Be?, in: Rand Journal of Economics, Vol. 21, 1990, S. 113-130.

[MeSz01] Messerschmitt D.G.; Szyperski, C.: Industrial and Economic Properties of Software: Technology, Processes, and Value; University of California at Berkeley; Computer Science Division Technical Report UCB//CSD-01-1130, Jan. 18, 2001, S. 1-51.

[Ordo91] Ordover, J. A.: A Patent System for Both Diffusion and Exclusion, in: Journal of Economic Perspectives, Vol. 5, Nr. 1,1991, S. 43-60.

[RiK100] Rivette, K.; Kline, D.: "Wie sich aus Patenten mehr herausholen lässt", in: Harvard Business Manager, Nr. 4, 2000, S. 28-40.

[ScRo90] Scherer, F.; Ross, D.: Industrial Market Structure and Economic Performance, Houghton Mifflin Company, Boston, 1990.

[Smet01] Smets-Solanes J.-P.: Stimulating Competition and innovation in the information society. Patents or sui generis right: what protection should be considered for software and other intangible innovations? Working document, draft version; http://www.proinnovation.org, Abruf am 2001-07-24.

[Tauch97] Tauchert, W.: Zur Beurteilung des technischen Charakters von Patentanmeldungen aus dem Bereich der Datenverarbeitung, Internet Zeitschrift für Rechtsinformatik, Web-Dok 27, 1997, Absatz 1-66, S. 1-19.

[ThRu99] Thompson, M. A.; Rushing, F. W.: An Empirical Analysis of the Impact of Patent Protection on Economic Growth: An Extension, in: Journal of Economic Development Vol. 24, Nr. 1,1999, S. 67-76.

[Weis88] Weisse, J.: Erfindungen, Patente, Lizenzen, 3. Aufl., Düsseldorf, 1988. 


\section{Appendix}

Um einen den erwarteten Barwert der Einzahlungsverbesserung durch Patentierung der in der aktuellen Periode gefundenen Innovation zu ermitteln, müssen die resultierenden erwarteten Einzahlungsüberschüsse (Formel 5) beider Alternativen über die Perioden der Patentlaufzeit verglichen und diskontiert (Zinssatz zs) werden.

$$
K W_{t}=\sum_{\tau=t}^{T_{P}} \frac{E^{p}\left[v_{i t+\tau}^{n e t}\right]-E^{o p}\left[v_{i t+\tau}^{n e t}\right]}{(1+z s)^{\tau}}=\sum_{\tau=1}^{T_{P}} \frac{\frac{v_{t+\tau}}{E^{p}\left[\sum_{i=1}^{I} x_{i t+\tau}\right]} \cdot p_{i t+\tau}-\frac{v_{t+\tau}}{E^{o p}\left[\sum_{i=1}^{I} x_{i+\tau}\right]} \cdot p_{i t+\tau}}{(1+z s)^{\tau}}
$$

Die eigene Innovationswahrscheinlichkeit $p_{\text {it }}$ wird zunächst als unabhängig von der Patentierungsentscheidung angenommen, während $\mathrm{v}_{\mathrm{t}}$ und $c_{i t}^{d}$, da exogen gegeben, in beiden Szenarien identisch sind.

Die Zahl der erwarteten Innovationen hängt von den jeweiligen durchschnittlichen Innovationswahrscheinlichkeiten ab, die im Falle der Patentierung negativ beeinflusst werden. Daraus ergibt sich folgender Zusammenhang:

$$
E^{p}\left[\sum_{i=1}^{I} x_{i t}\right] \leq E^{o p}\left[\sum_{i=1}^{I} x_{i t}\right] \quad 1 \leq E^{p}\left[\sum_{i=1}^{I} x_{i t}\right], E^{o p}\left[\sum_{i=1}^{I} x_{i t}\right] \leq I
$$

Aus Formel A1 folgt:

$$
\begin{aligned}
& B W=\sum_{\tau=1}^{T_{P}} \frac{\Delta E_{t+\tau}}{v_{t+\tau} \cdot p_{i t+\tau}\left(\frac{E^{o p}\left[\sum_{i=1}^{I} x_{i t+\tau}\right] \cdot\left(E^{o p}\left[\sum_{i=1}^{I} x_{i t+\tau}\right]-\Delta E_{t+\tau}\right)}{(1+z s)^{\tau-t}}\right)} \\
& \text { mit } \Delta E_{t+\tau}=E^{o p}\left[\sum_{i=1}^{I} x_{i t+\tau}\right]-E^{p}\left[\sum_{i=1}^{I} x_{i t+\tau}\right]
\end{aligned}
$$

Um die Differenz zwischen dem Erwartungswert ohne Patent und mit Patent $\Delta E$ zu bestimmen, muss die Differenz zwischen den jeweiligen durchschnittlichen Innovationswahrscheinlichkeiten ermittelt werden. 


$$
\begin{aligned}
& E_{t+\tau}\left[\sum_{i=1}^{I} x_{i t+\tau}\right]=\hat{p}_{t+\tau} \cdot I \quad E_{t+\tau}^{p}\left[\sum_{i=1}^{I} x_{i t+\tau}\right]=\hat{p}_{t+\tau}^{p} \cdot I \quad E_{t+\tau}^{o p}\left[\sum_{i=1}^{I} x_{i t+\tau}\right]=\hat{p}_{t+\tau}^{o p} \cdot I \\
& \Delta E_{\tau}=I \cdot\left(\hat{p}_{\tau}^{o p}-\hat{p}_{\tau}^{p}\right)=I \cdot \Delta p_{\tau}
\end{aligned}
$$

Zur Ermittlung der Wahrscheinlichkeitsdifferenz wird zunächst das relative Verhältnis ermittelt. Die einzige Komponente, die in den beiden Größen unterschiedlich ausgeprägt ist, ist die Abbildung der Wirkung früherer Patente anderer Akteure C (aus Formel 3).

$$
\begin{aligned}
& \frac{\hat{p}_{\tau}^{o p}}{\hat{P}_{\tau}^{p}}=\frac{C^{o p}}{C^{p}}=\varepsilon^{T_{P}-\tau+1} \rightarrow \Delta p_{t+\tau}=p_{t+\tau}^{o p} \cdot\left(1-\varepsilon^{T_{P}-\tau+1}\right) \rightarrow \\
& \Delta E_{t+\tau}=I \cdot \hat{p}_{t+\tau}^{o p} \cdot\left(1-\varepsilon^{T_{P}-\tau+1}\right) \text { und } E^{o p}\left[\sum_{i=1}^{I} x_{i t+\tau}\right]=I \cdot \hat{p}_{t+\tau}^{o p}
\end{aligned}
$$

Hieraus folgt, eingesetzt in die Barwertformel (A3), die Darstellung in Formel 8 (A6). Die eigene Innovationswahrscheinlichkeit in den Folgeperioden $p_{i t+\tau}$ wird approximiert durch die aktuelle $p_{i t}$.

$$
E\left[B W_{t}^{P Z}\right]=\sum_{\tau=1}^{T_{p}}\left(v_{t+\tau} \cdot p_{i t} \cdot \frac{\left(1-\varepsilon^{T_{P}-\tau+1}\right)}{I \cdot \hat{p}_{t+\tau}^{o p} \cdot \varepsilon^{T_{P}-\tau+1}} \cdot q^{\tau}\right)
$$

Analog zur Annahme in Gleichung 6 ist bei der Bildung von $\Delta E_{\tau}$ zu beachten, dass dieser Wert maximal $E^{o p}\left[\sum_{i=1}^{I} x_{i t+\tau}\right]-1$ annehmen kann, sofern nicht $E^{o p}\left[\sum_{i=1}^{I} x_{i t+\tau}\right]=E^{p}\left[\sum_{i=1}^{I} x_{i t+\tau}\right]$ ist. 